\title{
Robust Synchronization of Hyperchaotic Systems with Uncertainties and External Disturbances
}

\author{
Qing Wang, Yongguang Yu, and Hu Wang \\ Department of Mathematics, Beijing Jiaotong University, Beijing 100044, China \\ Correspondence should be addressed to Yongguang Yu; ygyu@bjtu.edu.cn
}

Received 4 December 2013; Revised 21 March 2014; Accepted 21 March 2014; Published 7 April 2014

Academic Editor: Zlatko Jovanoski

Copyright (C) 2014 Qing Wang et al. This is an open access article distributed under the Creative Commons Attribution License, which permits unrestricted use, distribution, and reproduction in any medium, provided the original work is properly cited.

The robust synchronization of hyperchaotic systems with uncertainties and external disturbances is investigated. Based on the Lyapunov stability theory, the appropriate adaptive controllers and parameter update laws are designed to achieve the synchronization of uncertain hyperchaotic systems. The robust synchronization of two hyperchaotic Chen systems is taken as an example to verify the feasibility of the presented schemes. The size of the subcontroller gain's influences on the convergence speed is discussed. Finally, numerical simulations are given to demonstrate the effectiveness of the proposed synchronization schemes.

\section{Introduction}

Since the method of synchronization between identical chaotic systems with different initial conditions was presented by Pecora and Carroll [1], chaos synchronization has attracted considerable attention because of its wide range of applications in many important research fields, such as secure communication, chemical reactions, artificial neural networks, and biological systems [2,3]. The idea of synchronization is to use the output of the drive system to control the response system so that the output of the response system follows the one of the drive system asymptotically. Up to now, many types of synchronization phenomena have been reported, such as generalized synchronization $[4,5]$, adaptive synchronization [6-9], projective synchronization [10-12], impulsive synchronization [13], lag synchronization [14, 15], and function projective synchronization $[16,17]$. And a wide variety of control approaches, such as backstepping design technique [18], fuzzy sliding mode control [19, 20], adaptive control [21], optimal control [22], and $H_{\infty}$ control [23], have been proposed to synchronize chaotic systems.

However, most of the reported schemes are mainly concerned with the synchronization of chaotic systems without uncertainties and external disturbances. This behaviour results in that the complexity of chaotic dynamics is limited while, in real world applications, there exist a mass of phenomena of the uncertainties and external disturbances in chaotic systems. In this regard, a number of researchers have paid their attention to the synchronization of chaotic systems with uncertainties and external disturbances [24-30]. The corresponding works have solved the problem of synchronization of chaotic systems with uncertainties and external disturbances. However, these studies have not considered the synchronization of the hyperchaotic systems.

The chaos-hyperchaos transition occurs when the second Lyapunov exponent becomes positive [31]. Compared with chaotic systems with one positive Lyapunov exponent, hyperchaotic systems are characterized by at least two positive Lyapunov exponents which indicates that they have more complex dynamics and much wider application. Moreover, the chaotic systems with higher-dimensional attractors have much more randomness and higher unpredictability of the corresponding system [32]. So the hyperchaos may be more useful in some fields such as communication and encryption. Motivated by this, robust synchronization of uncertain hyperchaotic systems is investigated. Aghababa [33] proposed finite-time chaos control and synchronization of fractionalorder nonautonomous chaotic (hyperchaotic) systems. In [34, 35], Fu considered robust adaptive modified function projective synchronization and robust adaptive antisynchronization of different hyperchaotic systems subject to external disturbances. Jawaada et al. [36] proposed robust active sliding mode antisynchronization of hyperchaotic systems with uncertainties and external disturbances. Li et al. [37] 
studied generalized function projective synchronization of two different hyperchaotic systems with unknown parameters. However, these studies have not considered the effects of both uncertainties and the different kinds of characteristics of external disturbances in the dynamics of the hyperchaotic systems.

In this paper, depending on the characteristics of external disturbance signals, appropriate adaptive controllers and parameter update laws are proposed for the robust synchronization of hyperchaotic systems with uncertainties and external disturbances. The uncertainties are bounded by a nonlinear state-dependent function, instead of a real constant. In the first adaptive controller, assuming that external disturbances are square integrable signals, the adaptivebased controllers and parameter update laws are designed to remove the effects of uncertainties. In the second proposed adaptive controller, the uncertainties and the external disturbances are not square integrable signals. The uncertainties and external disturbances are combined into an uncertain time-varying function with unknown bound. The robustness properties with respect to uncertainties and external disturbances are provided by the proposed controllers.

The organization of this paper is as follows. In Section 2, the robust synchronization of uncertain hyperchaotic Chen systems is formulated. According to the Lyapunov stability theory, the robust adaptive synchronization techniques and the error dynamical system's stability analysis are proposed in Section 3. Then, the simulation results in Section 4 are given to illustrate the effectiveness of the advised methods. Finally, the concluding remarks are given in Section 5.

\section{Problem Formulation}

Throughout the paper, for a vector $V \in R^{n},\|V\|$ denotes the Euclidean vector norm and $\|V\|_{Q}^{2}:=V^{T} Q V$, with the weighting matrix $Q$. Make $\|V\|^{2}:=\|V\|_{\mathrm{Q}=I}^{2}=V^{T} V$. Furthermore, $V \in L_{2}[0, T]$, if $\int_{0}^{T}\|V(t)\|^{2} d t<\infty, T \in[0, \infty)$.

Consider the nonlinear systems in the form of

$$
\begin{gathered}
\dot{X}=F(X), \\
X(0)=X_{0} \in R^{n}, \\
\dot{Y}=G(Y)+\Delta G+h+u, \\
Y(0)=Y_{0} \in R^{n},
\end{gathered}
$$

where $Y=\left[y_{1}, y_{2}, \ldots, y_{n}\right]^{T}$ and $X=\left[x_{1}, x_{2}, \ldots, x_{n}\right]^{T}$. The unstructured uncertainties are denoted as $\Delta G=$ $\left[\Delta G_{1}, \Delta G_{2}, \ldots, \Delta G_{n}\right]^{T}$ and the external disturbances are denoted as $h=\left[h_{1}, h_{2}, \ldots, h_{n}\right]^{T}$. The controller is $u=$ $\left[u_{1}, u_{2}, \ldots, u_{n}\right]^{T}$ which will be designed in the following section. The errors between system (1) and system (2) are denoted as $e_{i}=y_{i}-x_{i}(i=1,2, \ldots, n)$. And the error system is

$$
\dot{E}=G(Y)-F(X)+\Delta G+h+u,
$$

where $E=\left[e_{1}, e_{2}, \ldots, e_{n}\right]^{T}$.
The aim of the robust synchronization is to find an adaptive controller $u=\left[u_{1}, u_{2}, \ldots, u_{n}\right]^{T}$, such that the states of the nonlinear system (2) can follow the ones of the nonlinear system (1) asymptotically. Now, we adopt the adaptive control theory to achieve the robust synchronization of hyperchaotic Chen systems.

The hyperchaotic Chen system [38] is given by

$$
\begin{gathered}
\dot{x}=a(y-x)+w, \\
\dot{y}=d x-x z+c y, \\
\dot{z}=x y-b z, \\
\dot{w}=y z+r w,
\end{gathered}
$$

where $x, y, z$, and $w$ are state variables and $a, b, c, d$, and $r$ are the real constants.

When $a=35, b=3, c=12, d=7$, and $0 \leq r \leq 0.085$, system (4) is chaotic; when $a=35, b=3, c=12, d=7$, and $0.085<r \leq 0.798$, system (4) is hyperchaotic; when $a=35$, $b=3, c=12, d=7$, and $0.798<r \leq 0.9$, system (4) is periodic [38].

In order to observe the synchronization behavior of the hyperchaotic Chen systems, assume that there are two identical hyperchaotic Chen systems where the drive system with subscript 1 drives the response system having identical equations denoted by subscript 2 . For system (4), the drive system is

$$
\begin{gathered}
\dot{x}_{1}=a\left(y_{1}-x_{1}\right)+w_{1}, \\
\dot{y}_{1}=d x_{1}-x_{1} z_{1}+c y_{1}, \\
\dot{z}_{1}=x_{1} y_{1}-b z_{1}, \\
\dot{w}_{1}=y_{1} z_{1}+r w_{1},
\end{gathered}
$$

and the response system is

$$
\begin{gathered}
\dot{x}_{2}=a_{1}\left(y_{2}-x_{2}\right)+w_{2}+\Delta f_{1}\left(x_{2}, y_{2}, z_{2}, w_{2}\right) \\
+h_{1}(t)+u_{1}(t), \\
\dot{y}_{2}=d x_{2}-x_{2} z_{2}+c_{1} y_{2}+\Delta f_{2}\left(x_{2}, y_{2}, z_{2}, w_{2}\right) \\
+h_{2}(t)+u_{2}(t), \\
\dot{z}_{2}=x_{2} y_{2}-b_{1} z_{2}+\Delta f_{3}\left(x_{2}, y_{2}, z_{2}, w_{2}\right)+h_{3}(t)+u_{3}(t), \\
\dot{w}_{2}=y_{2} z_{2}+r_{1} w_{2}+\Delta f_{4}\left(x_{2}, y_{2}, z_{2}, w_{2}\right)+h_{4}(t)+u_{4}(t),
\end{gathered}
$$

where $a_{1}, b_{1}, c_{1}$, and $r_{1}$ are unknown parameters which need to be estimated; perturbation vectors are denoted as $\Delta f=\left[\Delta f_{1}, \Delta f_{2}, \Delta f_{3}, \Delta f_{4}\right]^{T}$ and $h=\left[h_{1}, h_{2}, h_{3}, h_{4}\right]^{T} ; \Delta f_{i}(i=$ $1,2,3,4)$ are the bounded unstructured uncertainties and $h_{i}(i=1,2,3,4)$ are the external disturbances. The controller vector is denoted as $u=\left[u_{1}, u_{2}, u_{3}, u_{4}\right]^{T}$. The synchronization errors are defined as $e_{1}=x_{2}-x_{1}, e_{2}=y_{2}-y_{1}, e_{3}=z_{2}-z_{1}$, and $e_{4}=w_{2}-w_{1}$. 
Subtract (5) from (6), error dynamical system can be represented in a matrix form as

$$
\dot{E}=A E+B \theta_{1}-B \theta+\Delta f+h+u,
$$

where $E=\left[e_{1}, e_{2}, e_{3}, e_{4}\right]^{T}, \theta_{1}=\left[a_{1}, b_{1}, c_{1}, r_{1}\right]^{T}$, and $\theta=[a, b$, $c, r]^{T}$. Consider

$$
\begin{aligned}
A & =\left(\begin{array}{cccc}
-a_{1} & a_{1} & 0 & 1 \\
d-z_{2} & c_{1} & -x_{1} & 0 \\
y_{1} & x_{2} & -b_{1} & 0 \\
0 & z_{1} & y_{2} & r_{1}
\end{array}\right), \\
B & =\left(\begin{array}{cccc}
y_{1}-x_{1} & 0 & 0 & 0 \\
0 & 0 & y_{1} & 0 \\
0 & -z_{1} & 0 & 0 \\
0 & 0 & 0 & w_{1}
\end{array}\right) .
\end{aligned}
$$

The aim of the robust synchronization is to design a controller vector $u=\left[u_{1}, u_{2}, u_{3}, u_{4}\right]^{T}$ such that the drive system (5) and the response system (6) are synchronized in the sense that $\lim _{t \rightarrow \infty}\|E(t)\|=0$.

\section{Robust Synchronization of Two Hyperchaotic Chen Systems}

In this section, depending on the characteristics of disturbance vector $h$ in (6), two adaptive controllers are designed to provide the robustness properties with uncertainties.

Two robust adaptive techniques are developed based on a control input in the form as

$$
u=-(A+K) E+B \theta-B \widehat{\theta}+u_{c 1}+u_{c 2}
$$

where $K=\operatorname{diag}\left(k_{1}, k_{2}, k_{3}, k_{4}\right)$ is a positive definite matrix and $k_{1}, k_{2}, k_{3}$, and $k_{4}$ are control gains. $\hat{\theta}=[\widehat{a}, \widehat{b}, \widehat{c}, \widehat{r}]^{T}$ are denoted as the estimated values of $\left[a_{1}, b_{1}, c_{1}, r_{1}\right]^{T}$. The parameter estimation errors vector is defined as $E_{n}=\left[e_{a}, e_{b}, e_{c}, e_{r}\right]^{T}$, where $e_{a}=a_{1}-\widehat{a}, e_{b}=b_{1}-\widehat{b}, e_{c}=c_{1}-\widehat{c}$, and $e_{r}=r_{1}-\widehat{r}$. $u_{c 1}$ and $u_{c 2}$ are denoted as the adaptive subcontrollers, which will be determined in the following theorems.

Assumption 1. The system external disturbances satisfy $h \in$ $L_{2}[0, \infty)$ and the unstructured uncertainties are bounded by a smooth nonlinear state-dependent function; that is, $\left\|\Delta f\left(x_{2}, y_{2}, z_{2}, w_{2}\right)\right\| \leq M\left(x_{2}, y_{2}, z_{2}, w_{2}\right)$.

Theorem 2. With Assumption 1, the robust synchronization between the drive system (5) and the response system (6) is realized by controller (9) with

$$
\begin{gathered}
u_{c 1}=-\frac{M^{2} E}{M\|E\|+\delta e^{-\sigma t}}, \\
u_{c 2}=-\frac{1}{2 \rho} E,
\end{gathered}
$$

and the parameter update laws

$$
\begin{gathered}
\dot{\hat{a}}=\left(y_{1}-x_{1}\right) e_{1}, \\
\dot{\hat{b}}=-z_{1} e_{3}, \\
\dot{\hat{c}}=y_{1} e_{2}, \\
\dot{\hat{r}}=w_{1} e_{4},
\end{gathered}
$$

where $\rho>0$ denotes the subcontroller gain; $\delta$ and $\sigma$ are positive constants.

Proof. Choose a Lyapunov function as

$$
V\left(E, E_{n}\right)=\frac{1}{2} E^{T} E+\frac{1}{2} E_{n}^{T} E_{n} .
$$

With the controller (9), the time derivative of (12) along the error trajectory of (7) is

$$
\begin{aligned}
\dot{V}= & -E^{T} K E+E_{n}^{T} B^{T} E+\Delta f^{T} E+h^{T} E \\
& +u_{c 1}^{T} E+u_{c 2}^{T} E+\dot{e}_{a} e_{a}+\dot{e}_{b} e_{b}+\dot{e}_{c} e_{c}+\dot{e}_{r} e_{r} .
\end{aligned}
$$

Substituting subcontrollers $u_{c 1}$ and $u_{c 2}$ from (10) implies that

$$
\begin{aligned}
\dot{V} \leq & -E^{T} K E+E_{n}^{T} B^{T} E+\left\|\Delta f^{T}\right\|\|E\|+h^{T} E-M\|E\| \\
& +\delta e^{-\sigma t}-\frac{1}{2 \rho} E^{T} E-\dot{\hat{a}} e_{a}-\dot{\widehat{b}} e_{b}-\dot{\hat{c}} e_{c}-\dot{\widehat{r}} e_{r} .
\end{aligned}
$$

Then, taking into account the update laws (11) and Assumption 1 , one can obtain

$$
\begin{aligned}
\dot{V} \leq & -E^{T} K E-\frac{1}{2 \rho}(E-\rho h)^{T}(E-\rho h) \\
& +\delta e^{-\sigma t}+\frac{1}{2} \rho\|h\|^{2} \\
\leq & -E^{T} K E+\delta e^{-\sigma t}+\frac{1}{2} \rho\|h\|^{2} .
\end{aligned}
$$

Now, the inequality (15) is integrated from $t=0$ to $t=T$, one can receive

$$
\begin{aligned}
\int_{0}^{T}\|E(t)\|_{K}^{2} d t \leq & V\left(E(0), E_{n}(0)\right)-V\left(E(T), E_{n}(T)\right) \\
& +\frac{\delta}{\sigma}\left(1-e^{-\sigma T}\right)+\frac{1}{2} \rho \int_{0}^{T}\|h(t)\|^{2} d t
\end{aligned}
$$

for all $0 \leq T<\infty$. The inequality (16) implies that $E(t)$ is square-integrable.

Besides, the external disturbance $h$ is bounded which means that there exists a $H>0$ such that $\|h\| \leq H$. Due to inequality (15), $\dot{V}$ can be bounded as $\dot{V} \leq-\lambda_{\min }\|E\|^{2}+\rho H^{2}+\delta$, where $\lambda_{\min }$ is the minimum eigenvalue of $K$. Choose $\lambda_{\min }>$ $\left(\rho H^{2}+\delta\right) / \varepsilon^{2}$ for any small $\varepsilon>0$. There exists $\eta=\lambda_{\min }-\left(\rho H^{2}+\right.$ $\delta) / \varepsilon^{2}>0$ such that $\dot{V} \leq-\eta\|E\|^{2}<0$ for all $\|E\|>\varepsilon$. Thus, there is $T>0$ such that $\|E\| \leq \varepsilon$ for all $t \geq T$. This implies that 
the synchronization error is uniformly ultimately bounded [39] and all the closed-loop signals are bounded. Because of the boundedness of all variables, $\dot{E}(t)$ in the error dynamical system (7) is also bounded. Therefore, according to Barbalat's lemma [39], one can conclude that $\lim _{t \rightarrow \infty}\|E(t)\|=0$. The robust synchronization of hyperchaotic systems (5) and (6) with uncertainties and external disturbances is achieved. The proof is completed.

Remark 3. Choosing a smaller $\rho>0$ causes the faster convergence speed of system (7). In fact, there exists a trade-off between the value of subcontroller gain $\rho$ and the magnitude of control input $u$.

Remark 4. To satisfy the smoothness of controller (9) without violating the convergence property of synchronization error, the exponential term in subcontroller $u_{c 1}$ formed by $\delta>0$ and $\sigma>0$.

Theorem 2 shows those disturbances which belong to $L_{2}[0, \infty)$. However, in some applications, this condition may not be satisfied for some disturbance signals. This fact motivates taking an effective scheme to solve the synchronization problem for this case. The following assumption is made to tackle such perturbations.

Assumption 5. In the uncertain response system (6), the unstructured uncertainties and the external disturbances are combined into one function, $\Delta g\left(x_{2}, y_{2}, z_{2}, w_{2}\right)=$ $\Delta f\left(x_{2}, y_{2}, z_{2}, w_{2}\right)+h(t)$ with $\|\Delta g\| \leq m$, where $m>0$ is an unknown parameter.

Theorem 6. With Assumption 5, the robust synchronization between the hyperchaotic systems (5) and (6) is ensured by controller (9) with $u_{c 1}=-\widehat{m}^{2}\left(E /\left(\widehat{m}\|E\|+\delta e^{-\sigma t}\right)\right), u_{c 2}=0$, and

$$
\dot{\hat{m}}=\varphi\|E\| \text {, }
$$

where $\widehat{m}$ is the estimate of $m ; \varphi>0$ denotes the adaptation gain.

Proof. Choose a Lyapunov function as

$$
V\left(E, E_{n}, \widetilde{m}\right)=\frac{1}{2} E^{T} E+\frac{1}{2} E_{n}^{T} E_{n}+\frac{1}{2 \varphi} \widetilde{m}^{2}
$$

where $\widetilde{m}=m-\widehat{m}$ denotes the estimation error. The time derivative of (18) along (7) can be received:

$$
\begin{aligned}
\dot{V}= & -E^{T} K E+E_{n}^{T} B^{T} E+\Delta f^{T} E+h^{T} E+u_{c 1}^{T} E \\
& +u_{c 2}^{T} E+\dot{e}_{a} e_{a}+\dot{e}_{b} e_{b}+\dot{e}_{c} e_{c}+\dot{e}_{r} e_{r}+\frac{1}{\varphi} \dot{\bar{m}} \widetilde{m} .
\end{aligned}
$$

Replacing $u_{c 1}$ and $u_{c 2}$, one can get

$$
\begin{aligned}
\dot{V} \leq & -E^{T} K E+E_{n}^{T} B^{T} E+\left\|\Delta f^{T}+h^{T}\right\|\|E\|-\widehat{\vec{m}}\|E\| \\
& +\delta e^{-\sigma t}-\dot{\hat{a}} e_{a}-\dot{\vec{b}} e_{b}-\dot{\vec{c}} e_{c}-\dot{\hat{r}} e_{r}-\frac{1}{\varphi} \tilde{m} \dot{\hat{m}} .
\end{aligned}
$$
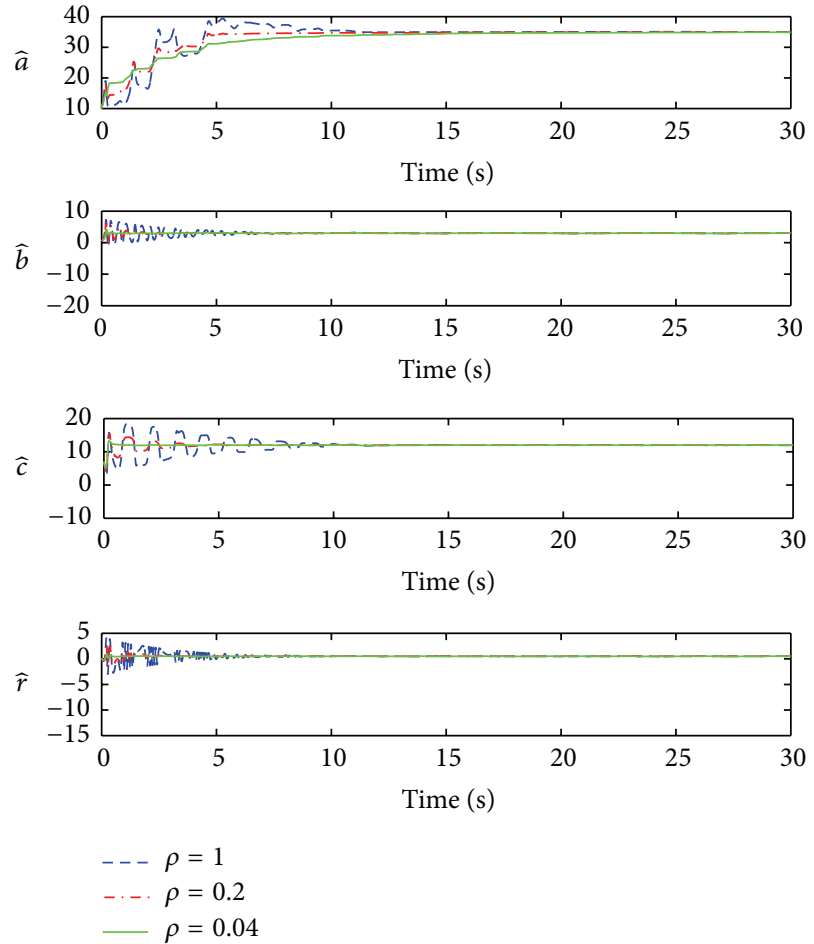

FIGURE 1: The estimated values of the unknown parameters $a_{1}, b_{1}, c_{1}$, and $r_{1}$.

Taking into account Assumption 5 and the parameter update laws (11), one can obtain

$$
\begin{aligned}
\dot{V} & \leq-E^{T} K E+\left\|\Delta g^{T}\right\|\|E\|-\widehat{m}\|E\|+\delta e^{-\sigma t}-\frac{1}{\varphi} \widetilde{m} \dot{\vec{m}} \\
& \leq-E^{T} K E+m\|E\|-\widehat{m}\|E\|+\delta e^{-\sigma t}-\frac{1}{\varphi} \widetilde{m} \dot{\bar{m}} .
\end{aligned}
$$

Substituting the update law (17) into inequality (21),

$$
\dot{V} \leq-E^{T} K E+\delta e^{-\sigma t} .
$$

Following a procedure, similar to the proof of Theorem 2, this implies that the goal of robust synchronization is achieved despite the systems (5) and (6) with time-varying parameters, the unstructured uncertainties, and external disturbances. The proof is completed.

\section{Numerical Simulations}

To verify and demonstrate the effectiveness of the proposed schemes, we will discuss the robust synchronization of the hyperchaotic Chen systems with different external disturbances of $h \in L_{2}[0, \infty)$ and $h \notin L_{2}[0, \infty)$, respectively. In the numerical simulations, the fourth-order Runge-Kutta method is used to solve the systems with time step size 0.001 .

The performance of the proposed robust adaptive synchronization schemes is demonstrated by the following two cases. For this numerical simulations, the system unstructured uncertainties are assumed to be $\Delta f_{i}=$ $0.02 \sin t \sqrt{x_{2}^{2}+y_{2}^{2}+z_{2}^{2}+w_{2}^{2}},(i=1,2,3,4)$ which results in 

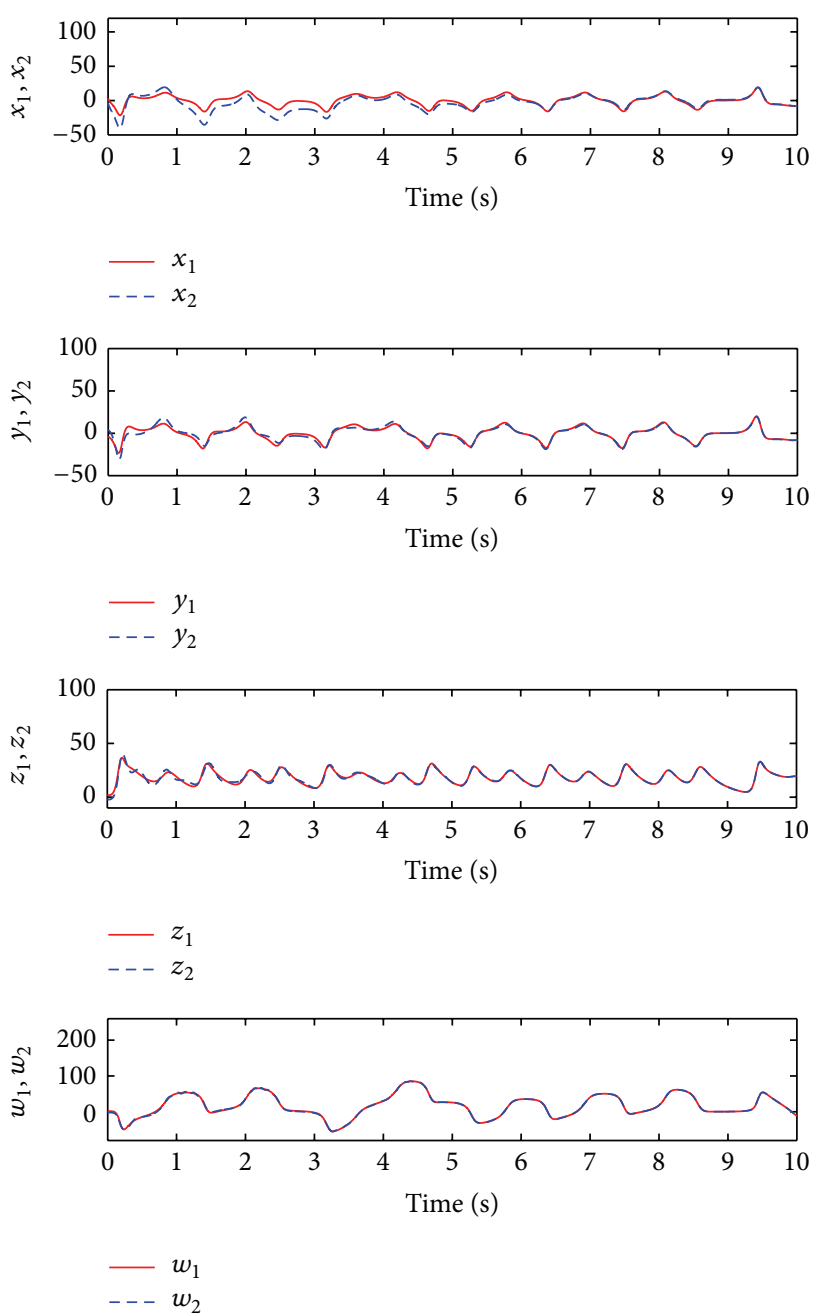

Figure 2: The states of the hyperchaotic Chen systems (5) and (6) with $\rho=1$.

$M=0.03 \sqrt{x_{2}^{2}+y_{2}^{2}+z_{2}^{2}+w_{2}^{2}}$. In each case, the parameters of the subcontroller $u_{c 1}$ are chosen as $\delta=0.01$ and $\sigma=0.01$.

Case I. Choose $a=35, b=3, c=12, d=7$, and $r=0.5$ and $a_{1}=35, b_{1}=3, c_{1}=12$, and $r_{1}=0.5$. The bounded and square-integrable disturbance signals $h_{i}=$ $0.01 e^{-t} \sin t,(i=1,2,3,4)$ perturb the response system (6). Take $k_{1}=0.1, k_{2}=0.1, k_{3}=0.1$, and $k_{4}=0.1$ and the initial conditions are $\widehat{a}(0)=10, \widehat{b}(0)=0.5, \widehat{c}(0)=7$, and $\widehat{r}(0)=0.1$. The initial states of the drive system (5) and response system $(6)$ are $\left(x_{1}(0), y_{1}(0), z_{1}(0), w_{1}(0)\right)=$ $(3,-4,2,2)$ and $\left(x_{2}(0), y_{2}(0), z_{2}(0), w_{2}(0)\right)=(-3,4,-2,-2)$, so the initial states of the error dynamical system (7) are taken as $\left(e_{1}, e_{2}, e_{3}, e_{4}\right)=(-6,8,-4,-4)$. The performance of synchronization is evaluated with subcontroller gains $\rho=1$, $\rho=0.2$, and $\rho=0.04$. The estimated values $\widehat{a}, \widehat{b}, \widehat{c}, \widehat{r}$ of the unknown parameters, plotted in Figure 1, show that the smaller the subcontroller gain $\rho>0$, the faster speed the estimated values $\widehat{a}, \widehat{b}, \widehat{c}$, and $\widehat{r}$ converge to $a_{1}=35, b_{1}=3$, $c_{1}=12$, and $r_{1}=0.5$. The states of the drive system (5) and
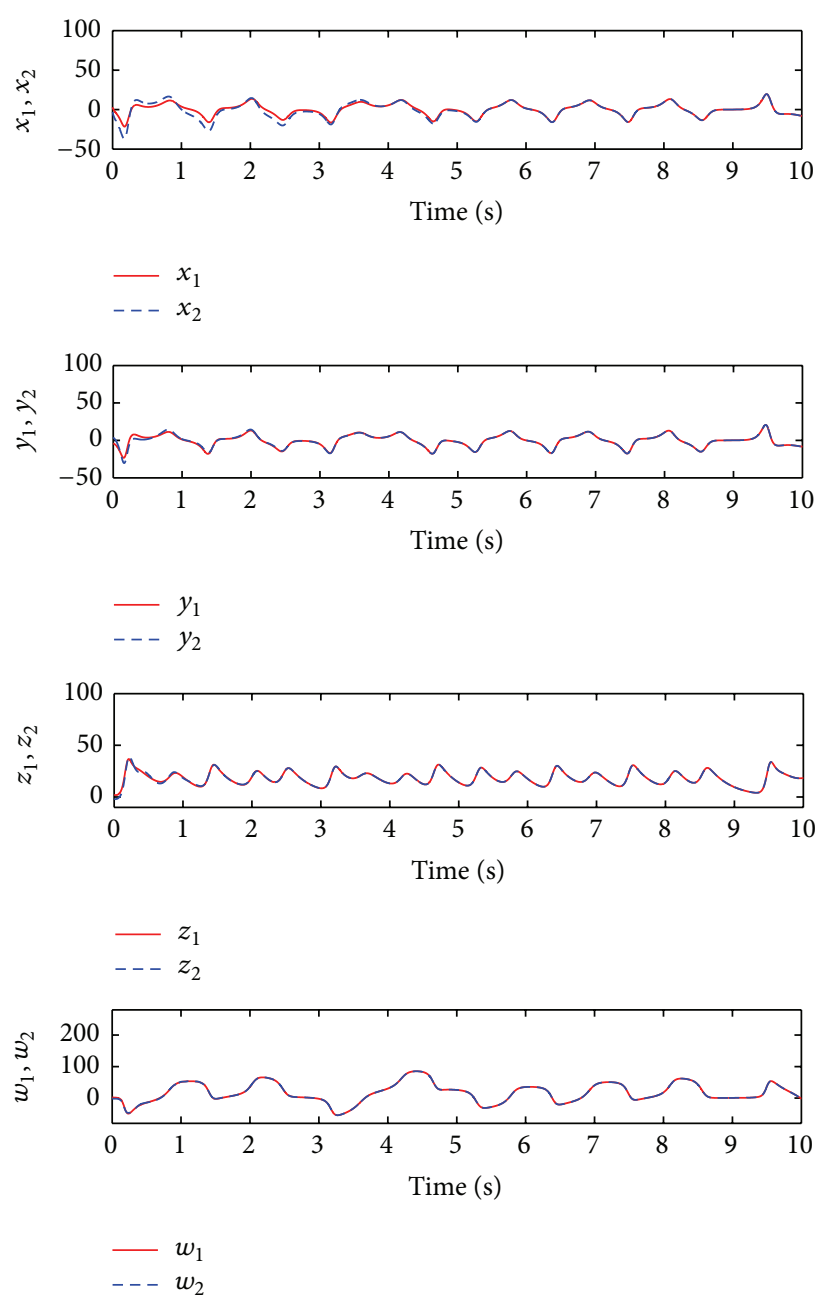

Figure 3: The states of the hyperchaotic Chen systems (5) and (6) with $\rho=0.2$.

the response system (6) with $\rho=1$ and $\rho=0.2$ are shown in Figures 2 and 3. The comparison of Figures 2 and 3 shows that the smaller the subcontroller gain $\rho>0$, the faster the speed of achieving synchronization. The states of the error dynamical system (7) with $\rho=1, \rho=0.2$, and $\rho=0.04$ in Figure 4 show that the smaller of the subcontroller gain $\rho>$ 0 provides the faster convergence speed of synchronization errors.

Case II. Take $a=35, b=3, c=12, d=7$, and $r=0.5$ and $a_{1}=35, b_{1}=3, c_{1}=12$, and $r_{1}=0.5$. As a general case, choose the disturbance signals as $h_{i}=0.02+0.02 \sin t,(i=$ $1,2,3,4)$ which do not belong to $L_{2}[0, \infty)$. Take $k_{1}=0.2$, $k_{2}=0.2, k_{3}=0.2$, and $k_{4}=0.2$ and the initial conditions are $\widehat{a}(0)=10, \widehat{b}(0)=0.5, \widehat{c}(0)=7, \widehat{r}(0)=0.1$, and $\widehat{m}(0)=0.01$. The initial states of the drive system (5) are $\left(x_{1}(0), y_{1}(0), z_{1}(0), w_{1}(0)\right)=(3,-4,2,2)$ and the initial states of the response system $(6)$ are $\left(x_{2}(0), y_{2}(0), z_{2}(0), w_{2}(0)\right)=$ $(-3,4,-2,-2)$. Hence the error dynamical system $(7)$ has the initial states $\left(e_{1}, e_{2}, e_{3}, e_{4}\right)=(-6,8,-4,-4)$. The estimated values $\hat{a}, \widehat{b}, \widehat{c}, \widehat{r}$ of the unknown parameters are shown 

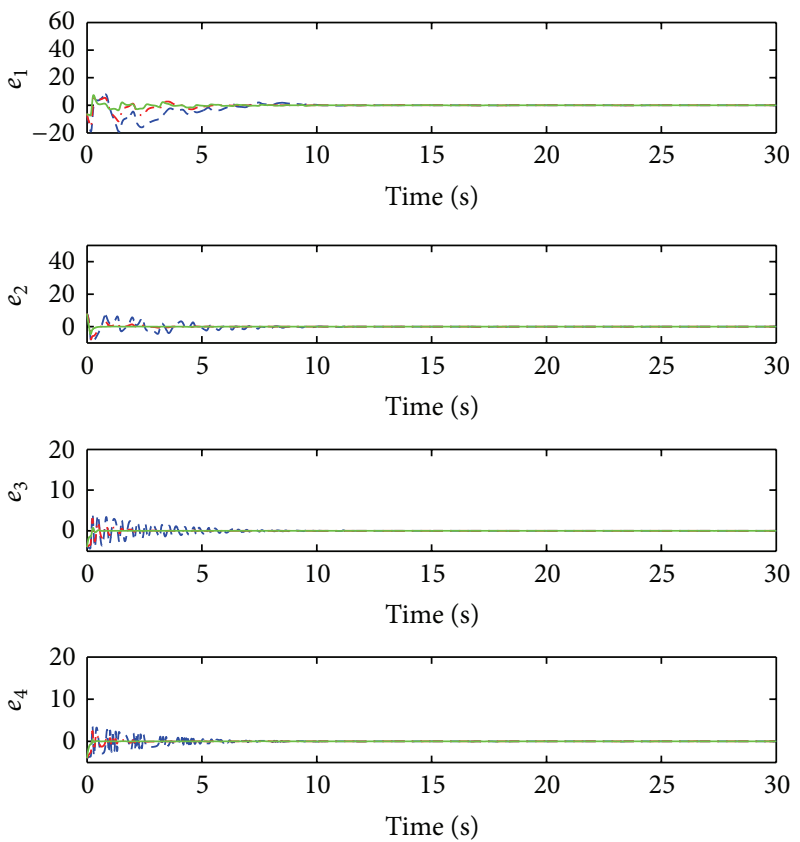

$$
\begin{aligned}
--\rho & =1 \\
--\rho & \rho=0.2
\end{aligned}
$$$$
\rho=0.04
$$

Figure 4: The states of the error dynamical system (7).

$\widehat{a}$
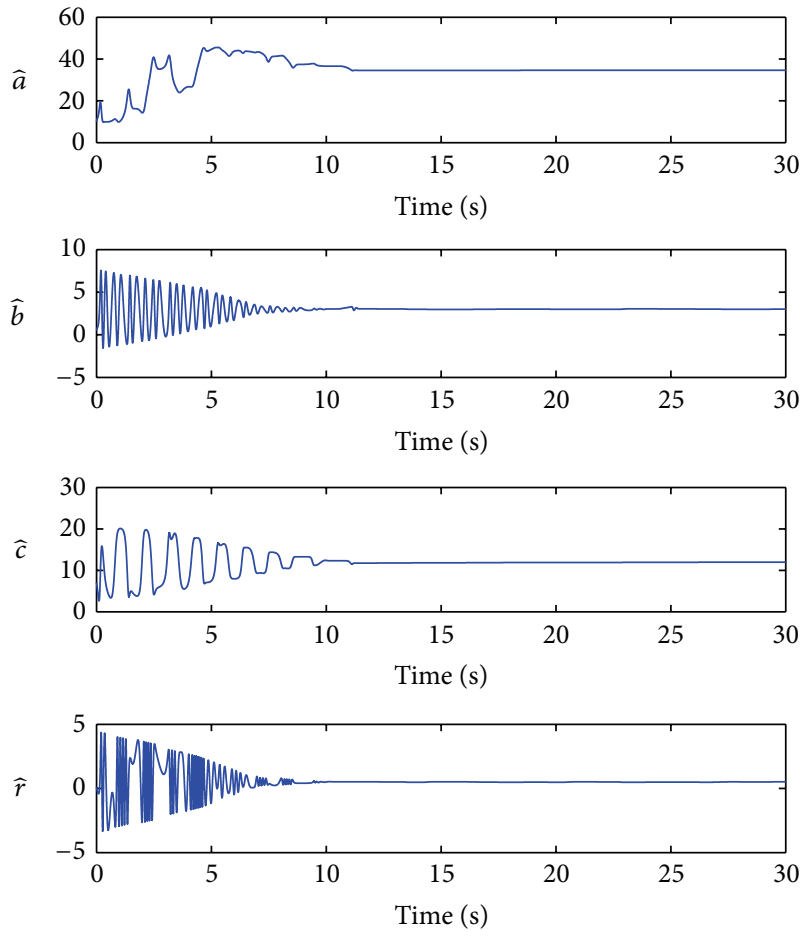

FIGURE 5: The estimated values of the unknown parameters $a_{1}, b_{1}$, $c_{1}$, and $r_{1}$.

in Figure 5. The states of the hyperchaotic Chen systems (5) and (6) are plotted in Figure 6. The convergence of synchronization errors are achieved, as depicted in Figure 7.

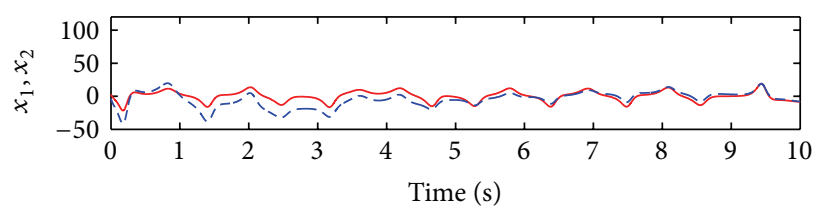

- $x_{1}$

-- - $x_{2}$
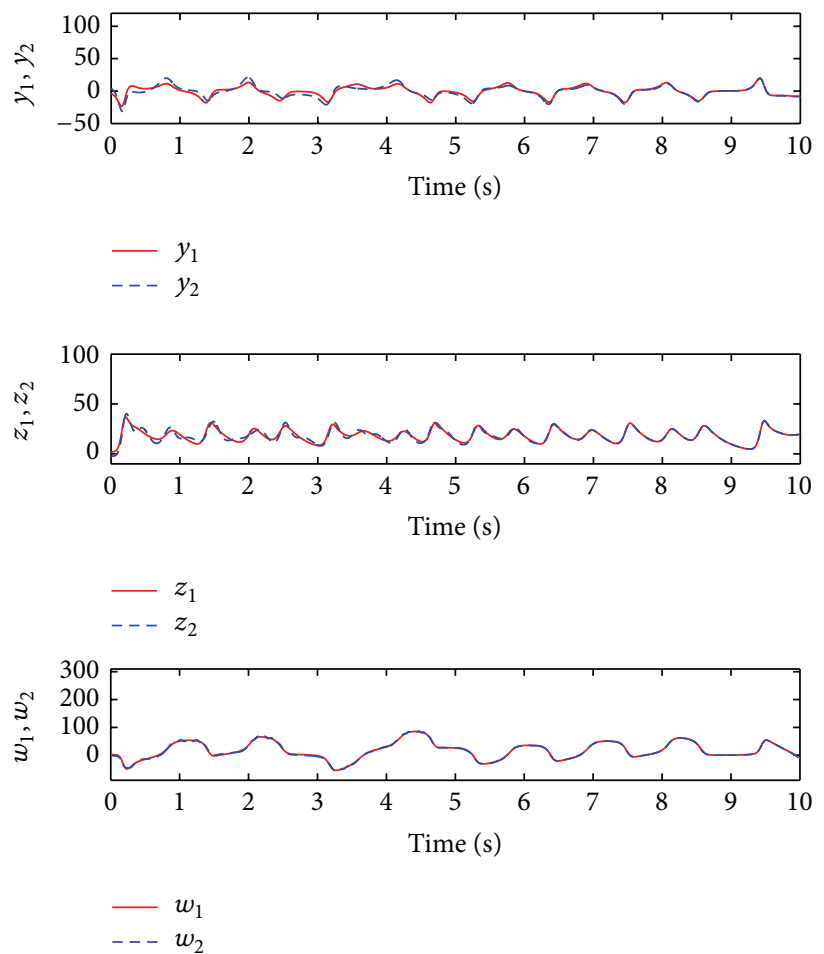

FIgURE 6: The states of the hyperchaotic Chen systems (5) and (6).
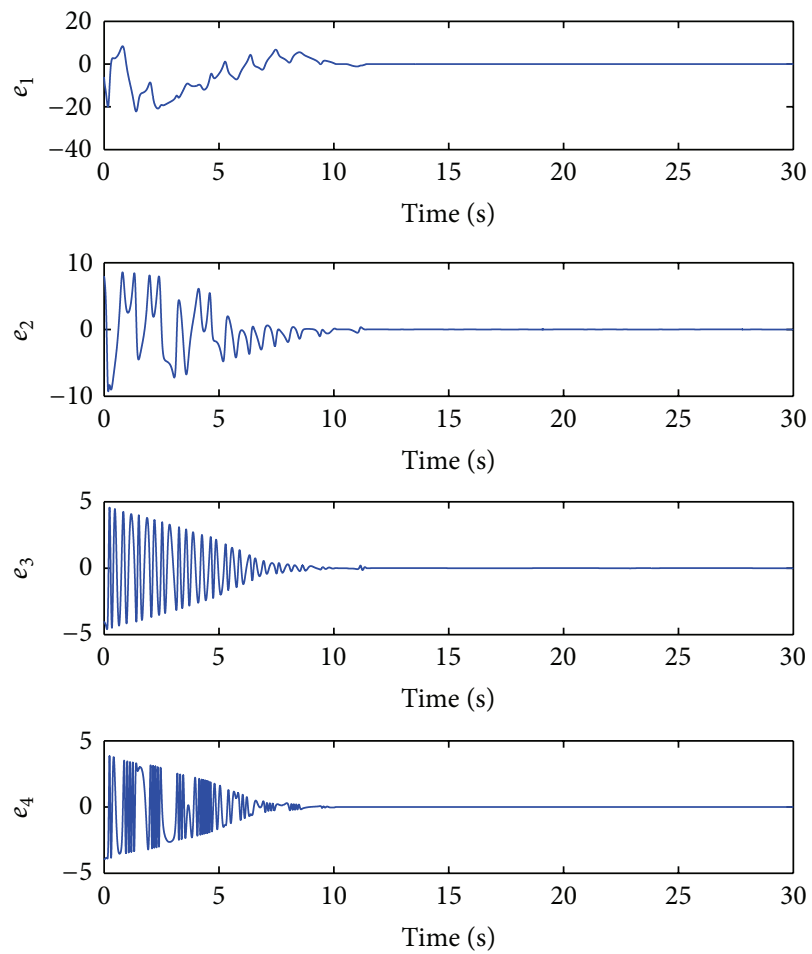

FIGURE 7: The states of the error dynamical system (7). 


\section{Conclusion}

In this paper, the robust synchronization of two hyperchaotic Chen systems with uncertainties and external disturbances is discussed. Depending on the different characteristics of disturbance signals $h \in L_{2}[0, \infty)$ or $h \notin L_{2}[0, \infty)$, two robust adaptive controllers are proposed. The appropriate adaptive controllers and the suitable parameter update laws are designed to remove the influence of uncertainties and external disturbances, so that robust synchronization of uncertain hyperchaotic Chen systems can be realized. The stability analysis is presented based on the Lyapunov stability theory. The smaller of the subcontroller gain causes the faster convergence speed of the system (7). At last, the efficiency and applicability of the introduced controllers and the parameter update laws are demonstrated using some numerical simulations.

\section{Conflict of Interests}

The authors declare that there is no conflict of interests regarding the publication of this paper.

\section{Acknowledgments}

This work is supported by the National Nature Science Foundation of China (no. 11371049) and the Science Foundation of Beijing Jiaotong University (no. 2011JBM130).

\section{References}

[1] L. M. Pecora and T. L. Carroll, "Synchronization in chaotic systems," Physical Review Letters, vol. 64, no. 8, pp. 821-824, 1990.

[2] G. Chen and X. Dong, From Chaos to Order: Methodologies, Perspectives, and Applications, vol. 24 of World Scientific Series on Nonlinear Science. Series A: Monographs and Treatises, World Scientific Publishing, River Edge, NJ, USA, 1998.

[3] L. M. Pecora and T. L. Carroll, "Driving systems with chaotic signals," Physical Review A, vol. 44, no. 4, pp. 2374-2383, 1991.

[4] X. Wang and Y. Wang, "Adaptive generalized synchronization of hyperchaos systems," International Journal of Modern Physics $B$, vol. 25, no. 32, pp. 4563-4571, 2011.

[5] Y. Yu, H.-X. Li, and J. Yu, "Generalized synchronization of different dimensional chaotic systems based on parameter identification," Modern Physics Letters B, vol. 23, no. 22, pp. 2593-2606, 2009.

[6] P. Liu and S. Liu, "Robust adaptive full state hybrid synchronization of chaotic complex systems with unknown parameters and external disturbances," Nonlinear Dynamics, vol. 70, no. 1, pp. 585-599, 2012.

[7] E. E. Mahmoud, "Adaptive anti-lag synchronization of two identical or non-identical hyperchaotic complex nonlinear systems with uncertain parameters," Journal of the Franklin Institute. Engineering and Applied Mathematics, vol. 349, no. 3, pp. 1247-1266, 2012.

[8] C.-C. Yang, "Adaptive synchronization of Lü hyperchaotic system with uncertain parameters based on single-input controller," Nonlinear Dynamics, vol. 63, no. 3, pp. 447-454, 2011.

[9] N. Smaoui, A. Karouma, and M. Zribi, "Adaptive synchronization of hyperchaotic chen systems with application to secure communication," International Journal of Innovative Computing, Information and Control, vol. 9, no. 3, pp. 1127-1144, 2013.

[10] G. M. Mahmoud and E. E. Mahmoud, "Modified projective lag synchronization of two nonidentical hyperchaotic complex nonlinear systems," International Journal of Bifurcation and Chaos, vol. 21, no. 8, pp. 2369-2379, 2011.

[11] Y.-A. Zheng, "Adaptive generalized projective synchronization of takagi-sugeno fuzzy drive-response dynamical networks with time delay," Chinese Physics Letters, vol. 29, no. 2, Article ID 020502, 2012.

[12] Z.-Y. Wu and X.-C. Fu, "Adaptive function projective synchronization of discrete chaotic systems with unknown parameters," Chinese Physics Letters, vol. 27, no. 5, Article ID 050502, 2010.

[13] C. Ma and X. Wang, "Impulsive control and synchronization of a new unified hyperchaotic system with varying control gains and impulsive intervals," Nonlinear Dynamics, vol. 70, no. 1, pp. 551-558, 2012.

[14] M. G. Rosenblum, A. S. Pikovsky, and J. Kurths, "From phase to lag synchronization in coupled chaotic oscillators," Physical Review Letters, vol. 78, no. 22, pp. 4193-4196, 1997.

[15] E. M. Shahverdiev, S. Sivaprakasam, and K. A. Shore, "Lag synchronization in time-delayed systems," Physics Letters A, vol. 292, no. 6, pp. 320-324, 2002.

[16] H. Du, Q. Zeng, C. Wang, and M. Ling, "Function projective synchronization in coupled chaotic systems," Nonlinear Analysis: Real World Applications, vol. 11, no. 2, pp. 705-712, 2010.

[17] H. Du, F. Li, and G. Meng, "Robust function projective synchronization of two different chaotic systems with unknown parameters," Journal of the Franklin Institute. Engineering and Applied Mathematics, vol. 348, no. 10, pp. 2782-2794, 2011.

[18] Y. Yu and H.-X. Li, "Adaptive hybrid projective synchronization of uncertain chaotic systems based on backstepping design," Nonlinear Analysis: Real World Applications, vol. 12, no. 1, pp. 388-393, 2011.

[19] C.-L. Kuo, "Design of a fuzzy sliding-mode synchronization controller for two different chaos systems," Computers \& Mathematics with Applications, vol. 61, no. 8, pp. 2090-2095, 2011.

[20] N.-S. Pai, H.-T. Yau, and C.-L. Kuo, "Fuzzy logic combining controller design for chaos control of a rod-type plasma torch system," Expert Systems with Applications, vol. 37, no. 12, pp. 8278-8283, 2010.

[21] W. Guo, S. Chen, and H. Zhou, "A simple adaptive-feedback controller for chaos synchronization," Chaos, Solitons \& Fractals, vol. 39, no. 1, pp. 316-321, 2009.

[22] J. M. V. Grzybowski, M. Rafikov, and J. M. Balthazar, "Synchronization of the unified chaotic system and application in secure communication," Communications in Nonlinear Science and Numerical Simulation, vol. 14, no. 6, pp. 2793-2806, 2009.

[23] S. M. Lee, D. H. Ji, J. H. Park, and S. C. Won, " $H_{\infty}$ synchronization of chaotic systems via dynamic feedback approach," Physics Letters A, vol. 372, no. 29, pp. 4905-4912, 2008.

[24] M. Srivastava, S. K. Agrawal, and S. Das, "Adaptive projective synchronization between different chaotic systems with parametric uncertainties and external disturbances," PramanaJournal of Physics, vol. 81, pp. 417-437, 2013.

[25] P. A. Mohammad and H. Feizi, "Design of a sliding mode controller for synchronizing chaotic systems with parameter and model uncertainties and external disturbances," Transactions of the Institute of Measurement and Control, vol. 34, pp. 990-997, 2012. 
[26] H. R. Koofigar, S. Hosseinnia, and F. Sheikholeslam, "Robust adaptive synchronization of uncertain unified chaotic systems," Nonlinear Dynamics, vol. 59, no. 3, pp. 477-483, 2010.

[27] M. P. Aghababa and A. Heydari, "Chaos synchronization between two different chaotic systems with uncertainties, external disturbances, unknown parameters and input nonlinearities," Applied Mathematical Modelling, vol. 36, no. 4, pp. 16391652, 2012.

[28] P. Liu and S. Liu, "Robust adaptive full state hybrid synchronization of chaotic complex systems with unknown parameters and external disturbances," Nonlinear Dynamics, vol. 70, no. 1, pp. 585-599, 2012.

[29] W. Jawaada, M. S. M. Noorani, and M. M. Al-Sawalha, "Active sliding mode control antisynchronization of chaotic systems with uncertainties and external disturbances," Journal of Applied Mathematics, vol. 2012, Article ID 293709, 14 pages, 2012.

[30] M. P. Aghababa and M. E. Akbari, "A chattering-free robust adaptive sliding mode controller for synchronization of two different chaotic systems with unknown uncertainties and external disturbances," Applied Mathematics and Computation, vol. 218, no. 9, pp. 5757-5768, 2012.

[31] T. Kapitaniak, K.-E. Thylwe, I. Cohen, and J. Wojewoda, "Chaos-hyperchaos transition," Chaos, Solitons \& Fractals, vol. 5, no. 10, pp. 2003-2011, 1995.

[32] X. Wu, H. Wang, and H. Lu, "Modified generalized projective synchronization of a new fractional-order hyperchaotic system and its application to secure communication," Nonlinear Analysis: Real World Applications, vol. 13, no. 3, pp. 1441-1450, 2012.

[33] M. P. Aghababa, "Finite-time chaos control and synchronization of fractional-order nonautonomous chaotic (hyperchaotic) systems using fractional nonsingular terminal sliding mode technique," Nonlinear Dynamics, vol. 69, no. 1-2, pp. 247-261, 2012.

[34] G. Fu, "Robust adaptive modified function projective synchronization of different hyperchaotic systems subject to external disturbance," Communications in Nonlinear Science and Numerical Simulation, vol. 17, no. 6, pp. 2602-2608, 2012.

[35] G. Fu and Z. Li, "Robust adaptive anti-synchronization of two different hyperchaotic systems with external uncertainties," Communications in Nonlinear Science and Numerical Simulation, vol. 16, no. 1, pp. 395-401, 2011.

[36] W. Jawaada, M. S. M. Noorani, and M. M. Al-Sawalha, "Robust active sliding mode anti-synchronization of hyperchaotic systems with uncertainties and external disturbances," Nonlinear Analysis: Real World Applications, vol. 13, no. 5, pp. 2403-2413, 2012.

[37] Z. Li and X. Zhao, "Generalized function projective synchronization of two different hyperchaotic systems with unknown parameters," Nonlinear Analysis: Real World Applications, vol. 12, no. 5, pp. 2607-2615, 2011.

[38] Z. Yan, "Controlling hyperchaos in the new hyperchaotic Chen system," Applied Mathematics and Computation, vol. 168, no. 2, pp. 1239-1250, 2005.

[39] M. Krstic, I. Kanellakopoulos, and P. Kokotovic, Nonlinear and Adaptive Control Design, John Wiley \& Sons, New York, NY, USA, 1995. 


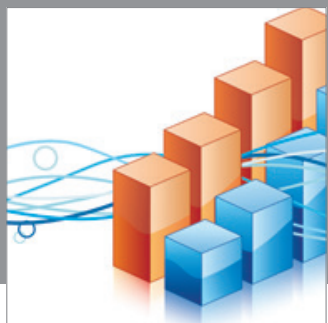

Advances in

Operations Research

mansans

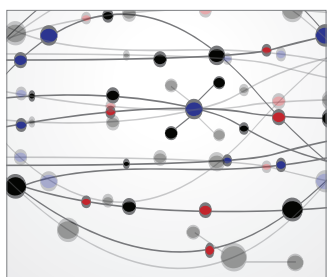

The Scientific World Journal
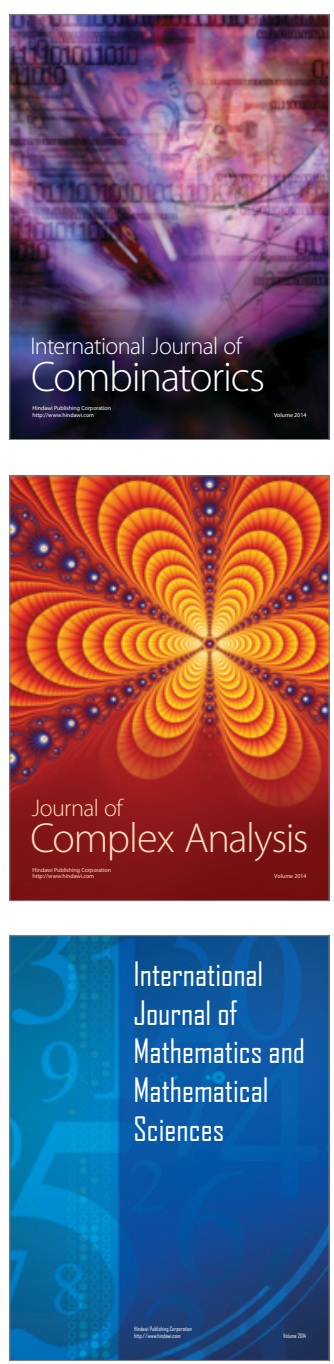
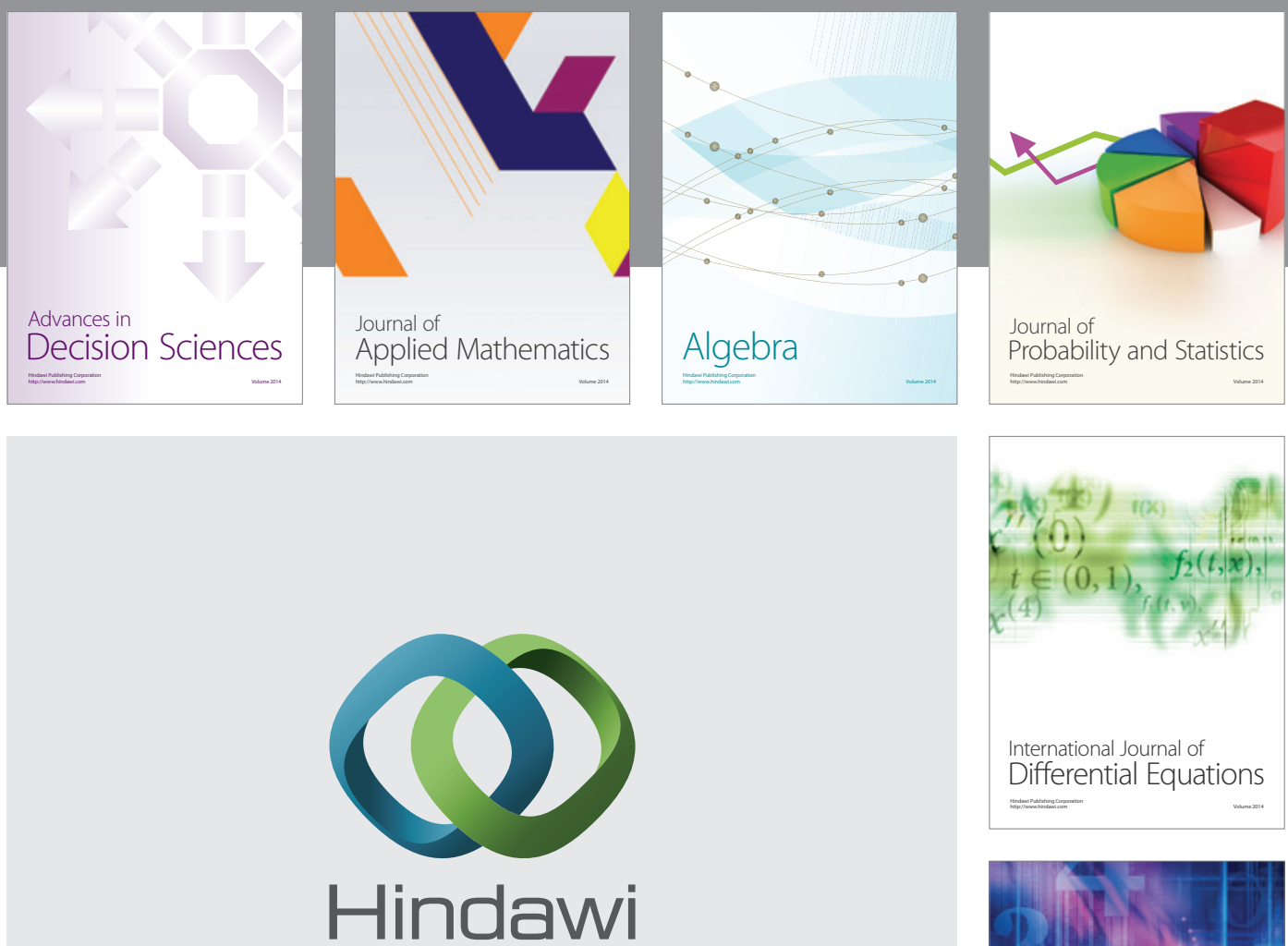

Submit your manuscripts at http://www.hindawi.com
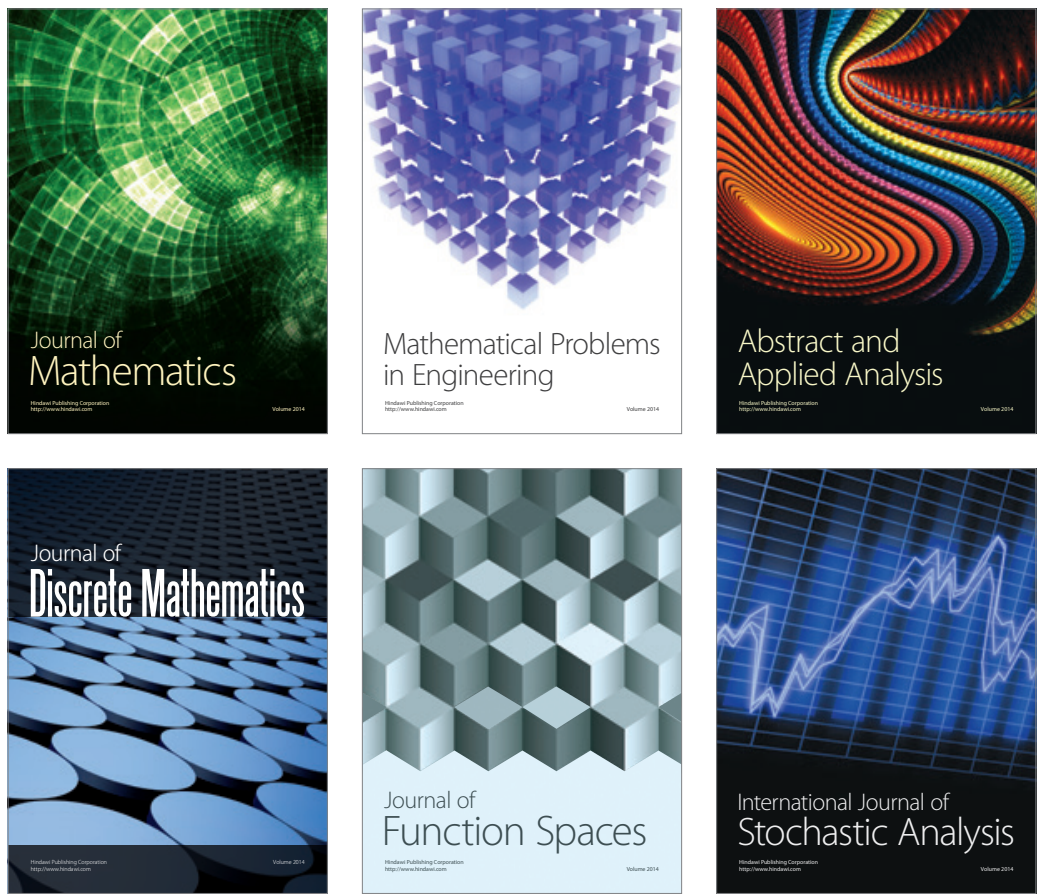

Journal of

Function Spaces

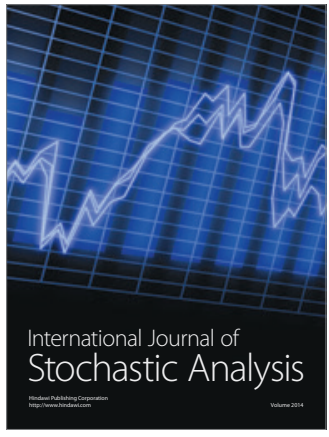

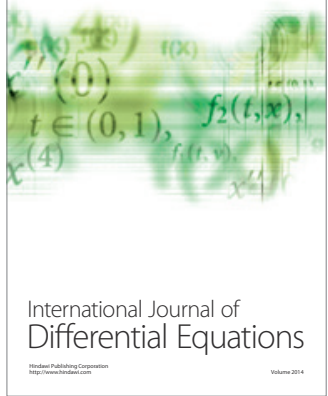
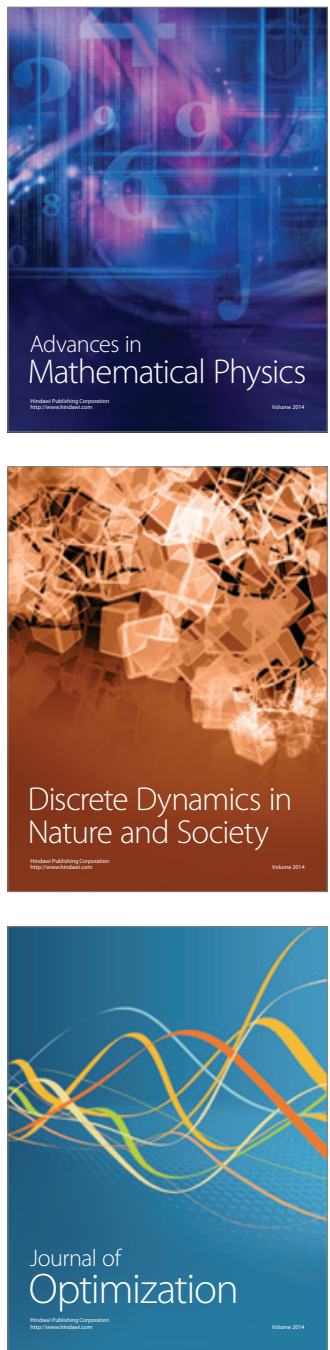